\title{
Introduction to the Public Health Section
}

This new section of the Journal of Headache and Pain is dedicated to an important sector of science: public health and the fields of headache, pain and other disorders. This section welcomes contributions that are relevant to persons who suffer from headache and pain disorders.

Although this may seem far from the concepts of public health, indeed the person and his environment as well as their interactions are the basis of public health.

In the healthcare sector, there is an emerging change that, I believe, is based on four pillars:

- The cultural change in the health sector is mostly based on the new concepts of health, functioning and disability introduced by the International Classification of Functioning, Disability and Health (ICF) of the World Health Organization. This first change deserves further investigation.

The second factor that is particularly relevant for chronic diseases has been given by the burden of diseases' approach: replacing mortality and morbidity indicators with disability indicators has given greater importance to the burden of neuropsychiatric disorders. In this regard, headache dis- orders rank among the first 20 diseases that cause years lived with disability (YLDs).

- The third issue that is relevant for health change is the trend in research. Research in fact is usually oriented and developed in three ways: basic, disease-oriented and patient-oriented research. This section places special attention on patient-oriented research and on its implications.

- Finally, another pillar of this Public Health section is the evaluation of the change from compliance to concordance in the relationship between patients and the healthcare system.

These points are object of this section, and contributions and discussion in this sense are welcomed. What does it mean to open a debate on science using instruments that usually are not considered to belong to science? This will need the definition of science as well as the integration of empirical with humanistic science to better serve the needs of science itself.

The first paper of this section introduces the main subject of our work: the person, starting from the biopsychosocial model and from the ICF definition that says that "Any person in any moment of life can 
have a health condition that in a negative environment becomes disability". The paper produced by the group at the Centre of Bioethics of Catholic University in Milan gives a brief overview on the latest concepts on health and suffering as well as on the open issues related to brain research.

Section Editor: Matilde Leonardi C. Besta Neurological Institute Via Celoria 11, I-20133 Milan, Italy e-mail:leonardi@istituto-besta.it 